\title{
Analysis of Transformer Vibration Signal
}

\author{
Keqi Ma \\ School of North China Electric Power University Baoding ,Baoding 071000, China \\ 2870662798@qq.com
}

Keywords: energy spectrum, vibration analysis

\begin{abstract}
In this paper, based on the measured signal, we use the least squares method to test the theoretical relationship between the vibration signal and current .Then, we use the Fourier transform data to analyze the relationship between amplitude and current .Finally, we analyze the relationship between energy and current of vibration signal by the definition of energy spectrum .This provides a reference for the vibration analysis of the operating state of the transformer.
\end{abstract}

\section{Introduction}

Power transformer power system is an important equipment which is essential for safe and reliable operation of the power system. Historical Statistics show that the transformer winding coil is one of the key parts of the fault[1-2]. Later, researchers have proposed a transformer-based vibration signal transformer core and winding condition monitoring technology[3-4]. A lot of researches have been done at home and abroad, mainly on the development of vibration signal acquisition system, the simulation of vibration generation mechanism and the processing technology of vibration signal[5]. Wang studied the relationship between fundamental frequency component of transformer box vibration and voltage, current and oil temperature, and based on which the vibration mathematical model of the box was put forward[6]. Ji proposed transformer vibration monitoring programs based on the load current law[7].

In this paper, the generation principle of the vibration signal of the power transformer, as well as the relationship between the vibration acceleration signal and the load current are analyzed. The relationship between the amplitude and the current is analyzed. The definition of the energy spectrum and its applicable range are introduced. The mathematical model of the vibration signal 、 vibration signal energy and voltage and current of the transformer is presented. The parameters of the mathematical model are calculated by the field test data. And thus examined the relationship between the vibration signal and the energy of the vibration signal and analyzed the influence of current on the amplitude after Fourier transform .

\section{The basic principle of transformers surface vibration signals}

\subsection{The generation and transmission of Power Transformer vibration}

The vibration of power transformer is mainly caused by the vibration of body structure and cooling device[8], where the body structure refers to the power transformer winding and core. The core vibration of the power transformer is mainly caused by the electromagnetic attraction, which is caused by the magnetostriction of the silicon steel sheet. The vibration of the winding is mainly caused by the load current passed through the winding.

2.2 Analysis of Relationship Between Vibration Signal and Voltage[9]

Assume the supply voltage $U_{1}=U_{s} \sin \omega$. According to the principle of electromagnetic Induction, the agnetic induction generated in the core can be expressed as

$$
B=\frac{\varphi}{S}=\frac{U_{s}}{\omega N S} \cos \omega t=B_{0} \cos \omega t
$$

Where ${ }_{\varphi}$ is core magnetic flux, $S$ is core ross-sectional area. Since the magnetic flux density and 
magnetic field strength of the linear relationship, the magnetic field strength in the core is $H=B / \mu=B H_{c} / B_{s}=B_{0} H_{c} \cos \omega / B_{s}$

Where $B_{s}$ is core saturation magnetic inducti $H_{c}$ is coercivity。

Under the action of external magnetic field, silicon steel sheet by the magnetostriction of the small deformation to meet the following relationship

$$
\left.\begin{array}{l}
\varepsilon=\Delta L / L \\
\frac{\Delta L}{L} \frac{1}{d H}=|H| \frac{2 \varepsilon_{s}}{H_{c}^{2}}
\end{array}\right\}
$$

Based on the above relationships, the maximum axial expansion and contraction of the core silicon steel sheet caused by magnetostriction is:

$$
\begin{aligned}
& \Delta L=L \int_{0}^{H}|H| \frac{2 \varepsilon_{s}}{H_{c}^{2}} d H=L \frac{\varepsilon_{s} H^{2}}{H_{c}^{2}}= \\
& L \frac{\varepsilon_{s} B^{2}}{B_{c}^{2}}=\frac{L \varepsilon_{s} U_{s}^{2}}{\left(\omega N_{\text {core }} S B_{s}\right)^{2}} \cos ^{2} \omega t
\end{aligned}
$$

It can be obtained when the transformer no-load, the silicon steel sheet magnetostriction caused by vibration acceleration of the core

$$
a_{c}=\frac{d^{2} \Delta L}{d t^{2}}=-\frac{2 L \varepsilon_{s} U_{s}^{2}}{\left(N_{\text {core }} S B_{s}\right)^{2}} \cos 2 \omega t
$$

By the formula (5), Vibration acceleration in the core material, operating temperature and other conditions of the same situation is proportional to the square of the voltage

$$
a_{c} \propto u_{s}^{2}
$$

2.3 Analysis of the Relation Between Vibration Signal and Current

When the load winding current in the transformer winding, due to the presence of leakage magnetic field, the winding between the dynamic electromagnetic force, causing the winding vibration. Suppose the current flowing through the windings in a stable power transformer then the role of the electromotive force on the winding coil is

$$
\begin{gathered}
I=I_{m} \sin \left(\omega t+\phi_{0}\right) \\
F=p I_{m}^{2}\left(\frac{1}{2} \cos \left(2 \omega t+2 \phi_{0}\right)-\frac{1}{2}\right)
\end{gathered}
$$

According to the dynamics theorem, the differential equation of winding displacement is obtained as follows:

$$
M \frac{d^{2} z}{d t^{2}}+C \frac{d z}{d t}+K z=F+M g
$$

Solving this equation, we can get the winding acceleration.

From the theoretical analysis, $a_{w}$ can be obtained.

$$
a_{w} \propto I_{m}^{2}
$$

The vibration of the transformer winding consists of a steady-state component and a gradually attenuating component, So in the ideal state ,the transformer winding vibration acceleration signal is also twice the current frequency $(\approx 100 \mathrm{~Hz})$

\section{Definition and Application of Energy spectrum}

The Energy spectral density is defined as:

$$
G(f)=|S(f)|^{2}(J / H z)
$$


where $S(f)$ is the Fourier transform of $s(t)$.

Energy

$$
E=\int_{-\infty}^{+\infty} G(f) d f
$$

Since $S(f)$ is even function,

$$
E=2 \int_{0}^{\infty} G(f) d f
$$

From the definition of Fourier transform and energy spectrum, we assume that the energy of the vibration signal is proportional to the fourth power of the voltage and the fourth power of the current.

\section{The acquisition of vibration signal on transformer surface}

The vibration signal acquisition system includes a sensor, a data acquisition instrument and a PC. Acceleration sensor testing using ULT2008 ICP acceleration sensor, data acquisition instrument using NI's data acquisition and analysis products.

In this paper, $110 \mathrm{KV}$ power transformer on-site testing. Acquisition parameters set: sampling frequency $10 \mathrm{KHz}$, each sampling time 1 s, every $5 \mathrm{~min}$ sampling time, the total continuous sampling 20h.
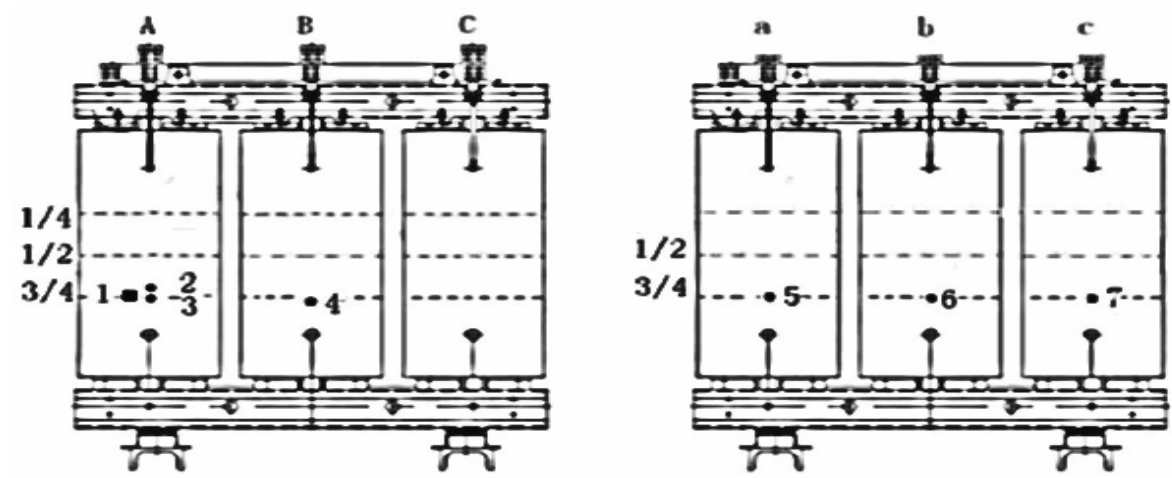

Fig 1. Recommended sensor positions on the transformer surface

5. Normalization processing of voltage and current

$$
I_{n}=\frac{i(n)}{I_{\text {average }}}
$$

( $I_{\text {average }}$ is the average current, $i(n)$ for each moment current)

\section{6.least squares}

Assuming:

$$
\begin{gathered}
{[\alpha, \beta, \gamma]=A} \\
{\left[X_{1}, X_{2}, X_{3}\right]=X} \\
{\left[Y_{1}\right]=Y}
\end{gathered}
$$

Solve the parameters

$$
\begin{gathered}
\|\bar{A} X-Y\|=\|A X-Y\|_{\text {Din }} \\
\bar{A}=Y X^{\mathbf{T}}\left(X X^{\mathbf{T}}\right)^{-\mathbf{1}}
\end{gathered}
$$

\section{The Relationship Between Vibration Signal and Current}

As the signal acquisition process, the voltage change is not large, the current change is large, therefore, consider the relationship between amplitude and current.

Theoretically, the vibration acceleration signal is proportional to the square of the current.By the 
paper, We use this formula to verify the quadratic relationship between the vibration signal and the square of the current.

As can be seen from the image, there is a big gap between the predicted value and the measured value. Since the change of the impact of vibration signal factors, including voltage, temperature is very small,therefore, in practice, can not be considered vibration signal amplitude and the square of the current is proportional
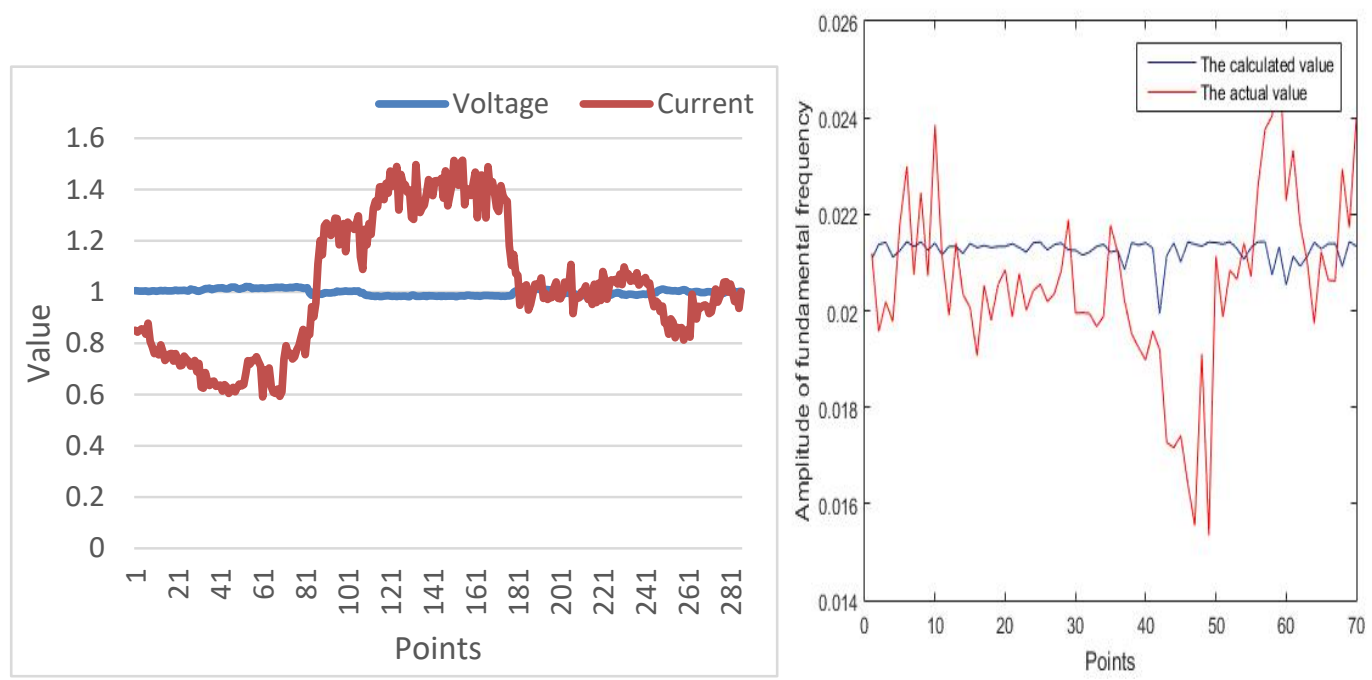

Fig.2

\section{The relationship between amplitude and current}

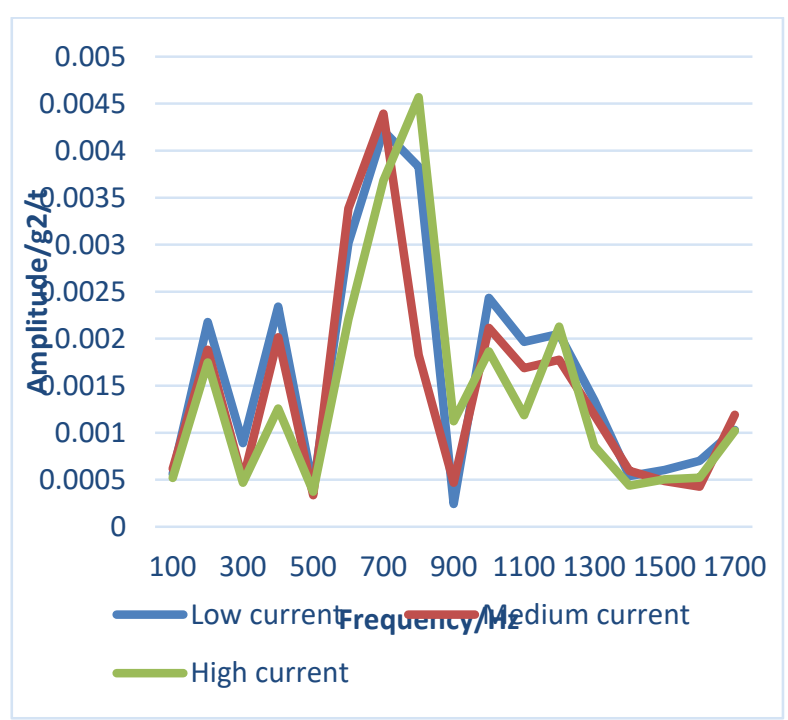

Fig.3

Since the signal by Fourier transform, the amplitude is concentrated in $2000 \mathrm{~Hz}$, take 100 to $1700 \mathrm{~Hz}$ of data, as follows,

It can be seen from the figure, the maximum amplitude after the Fourier transform is concentrated around $700 \mathrm{~Hz}$,so ,the original theory that frequency amplitude accounts for a large proportion can not be established. Even, the fundamental frequency amplitude is very small .

When the current is high, medium and low, the change of the amplitude is basically the same. Indicating that the current change has little effect on the trend and magnitude of the amplitude. 


\section{Analysis and Application of Energy Spectrum}

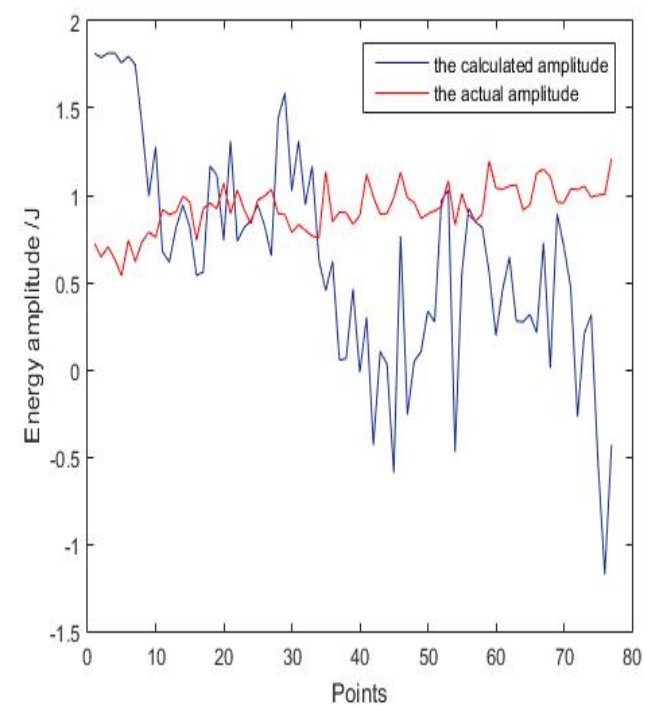

Fig. 4

According to the energy calculation formula, we obtain that the energy of the vibration signal is more closely related to the fourth power of the current and the fourth power of the voltage.Compare the current quadratic polynomial and the energy of the vibration signal ,use the least squares method to obtain the parameters, and to predict energy value, as follows:

As can be seen from the image, there is a big gap between the predicted value and the measured value. Since the change of the impact of vibration signal factors, including voltage, temperature is very small,therefore, in practice, can not be considered vibration signal amplitude and the square of the current is proportional.

\section{Summary}

The comparison between the predicted value and the actual value of the vibration signal shows that the relationship between the vibration signal and the square of the current is not true in the actual operation of the transformer. Since the voltage change is small during the operation of the transformer, the relationship between vibration signal and voltage can not be tested.

The change of current does not affect the trend of amplitude after Fourier transform, and will not affect the magnitude of amplitude .The amplitude after Fourier transform is concentrated near 700 $\mathrm{Hz}$, and the values at other frequencies are relatively small. The fundamental frequency amplitude is very small , this is contrary to the original conclusion.

This indicates that the linear relationship between the energy of the vibration signal and the fourth power of the current does not hold.

\section{References}

[1] LING Min, GU Zhengxian. Nation-wide fault statistics and analysisof transformers from 1990 to 1994 [J]. Power System Technology, 1996, 20(9): 47—52.

[2] WANG Mengyun - Nation-wide fault statistics and analysis of $110 \mathrm{kV}$ transformers[J. Distribution \& Utilization, 2006, 23(1): 1-4.

[3] SOKOLOV V. Effective methods of assessment of insulation system conditions in power transformers: a view based on practical experience [C] Proceeding of the 1999 CIGRE Regional Meeting. Paris, France: CIGRE, 1999: 659-667.

[4] LAVALLE J C. Failure detection in transformer using vibrational analysis[D]. Cambridge, 
MA, USA: MIT,1986.

[5] JI Shengchang, LIU Weiguo, SHAN Ping, et a1. The vibration measuring system for monitoring core and winding condition of power transformer [J]. High Voltage Engineering, 2000, 26(6): I-3.

[6] ARTOLETTU C, DESIDERIO M, FAZIO G, et a1. Vibro-acoustic techniques to diagnose power trans_-formers[J]. IEEE Transactions on Power Delivery, 2004,19(1):221-229.

[7] JI Shengchang, LI Yanming, FU Chenzhao, et a1. Application of on-load current method in monitoringThe condition of transformers core based On the vibration analysis method [J]. Proceedings of the CSEE, 2003, 23(6): 154-158.

[8] HYVARINEN A, KARHUNEN J, QIA E. Inde-pendent component analysis [M]. New York, USA:John Wiley 8L Sons Inc.2001: 96-128.

[9] Yeye.JI Shengchang.ZHANG Fan.LIU Yong.DONG Hongkui.CUI Zhigang.WU Jiawei. Power transformer vibration mechanism and influencing factors [Papers] -Journal of Xi'an Jiaotong University 2015 (6).

[10] MA Fujian. XU Jian. CHEN Keliang.WANG Fenghua. JIANG Yiming .JIN Zhijian Transformer on-line monitoring based on vibration analysis [Papers] - East China Power 2009 (7). 